\title{
Targeting efficiency of immunliposome; quantitative assessment
}

\begin{abstract}
Immuno liposomes (ImLp) are important tool in specific drug targeting and delivery. They are prepared by tagging antibodies onto the surface of liposomes enabling them to recognize specific antigen bearing cells so that drug can be delivered in targeted manner. However, no method is available to evaluate the accessibility and optimal orientation of antibodies borne by the ImLp. They are generally estimated in terms of protein, which provides no information regarding their number and availability and also no clue about how much ImLp would be sufficient for effective discrimination of target antigen. Optimal antibody density and its presentation are crucial for preventing the nonspecific association of ImLp that may lead to side effects. Here a method is presented to estimate the appropriately oriented antibodies on any type of ImLp. This information is vital for dose related decisions as both selective discerning by ImLp and the amount of encapsulated drug are equally important for the success of targeted drug therapy.
\end{abstract}

Keywords: immunoliposome, antibody targeting, drug delivery, antibodies, ELISA
Volume 2 Issue 2 - 2016

\author{
Ranjna C Dutta \\ Department of Biochemistry, Northwestern University, USA
}

Correspondence: Ranjna C Dutta, ExCel Matrix Biological Devices P Ltd., 12-5- 149/16,Vijayapuri, Tarnaka, India, Email ranjna_dutta@rediffmail.com

Received: October 07, 2015 | Published: February 12, 2016
Abbreviations: ImLp, immunoliposome; LDH, lactate dehydrogenase; BWW, biggers whitten and whittingham; SDS, sodium dodecyl sulfate; TBS, tris buffer saline; PBS, phosphate buffer saline; PC, phosphatidyl choline; DPPE, dipalmitoyl phosphatidyl ethanol amine; NHSO, N-hydroxy succinimide oleate

\section{Introduction}

Immunoliposomes ( $\operatorname{ImLp}$ ) have shown great promise in targeted drug delivery/release with enormous significance in the treatment of terminal diseases like cancer. ${ }^{1,2}$ Methods for their preparation and application in drug targeting are now well established. ${ }^{3-5}$ However, since no method is available for quantifying the targeting efficiency of Immunoliposome, batch-to-batch variation is common. As a consequence therapeutic efficacy of $\operatorname{ImLp}$ is also inconsistent. The antibodies on ImLp are usually estimated by its protein content that offers no clue as to how many antibodies are appropriately presented and therefore factually available for antigen recognition and binding. When the antibodies used for ImLp synthesis are purely monoclonal and tailored for upright liposome incorporation, protein estimation may be sufficient, but most often this is not the case. This causes substantial batch variation in terms of targeting efficiency of ImLp and in turn influences the dose for desired therapeutic outcome. Considering the fact that protein content is not the true reflection of antigen recognition ability of $\operatorname{ImL}$, quantification of appropriately oriented antibodies in a given preparation of $\operatorname{ImLp}$ is attempted. ELISA protocol is adapted for measuring $\operatorname{ImLp}$ titre and this information is subsequently used for calculating percentage of appropriately oriented antibodies.

\section{Materials and method}

Partially purified antibodies [RbamC4 (AS cut), 1:16,000] raised against testis specific lactate-dehydrogenase from mouse $\left(\mathrm{mLDHC}_{4}\right)$ in rabbit are used for the preparation of ImLp. All the chemicals including Biggers, Whitten and Whittingham (BWW) medium, Triton X-100, Tween-20, sodium dodecyl sulfate (SDS), phosphate buffer saline (PBS), tris buffer saline (TBS), cholesterol, egg phosphatidyl choline (PC), dipalmitoyl phosphatidyl ethanol amine (DPPE), N-hydroxysuccinimide oleate (NHSO), 1-ethyl-3-(3-dimethylaminopropyl)-carbodiimide (EDAC), bovine serum albumin (BSA), HRP-conjugated goat-anti-rabbit antibody and 2,2'-azino-bis-3- ethylbenzthiazoline-6-sulfonic acid (ABTS) are purchased from SIGMA. Carboxy-fluorescein (6-CF) (mol wt 376.32) single isomer is purchased from Molecular Probes. Immuno-certified plates for ELISA are purchased from Costar (Cambridge, MA, USA).

\section{Preparation of uni-lamellar liposome}

Small uni lamellar liposomes (0.05-0.10 micron size) incorporating $\mathrm{CF}(0.2 \mathrm{M})$ as a marker are prepared by the standard method of film hydration and sonication under $\mathrm{N}_{2}$ atmosphere. ${ }^{6}$ To eliminate the un-encapsulated marker as well as unused lipids the liposomes are passed through a Sephadex G-50 column $(19 \times 1 \mathrm{~cm})$, packed and equilibrated with tris buffer saline (TBS, $\mathrm{pH}$ 7.4). Liposome rich fractions are pooled and divided into two portions; one is used as control liposome and the other for the preparation of ImLp.

\section{Preparation of Immuno liposome (ImLp)}

Antibodies $\left(\mathrm{mLDHC}_{4}\right)$ are covalently attached to the surface of liposome through NHSO using EDAC. ${ }^{7}$ ImLp preparation is passed through another Sephadex G-50 column $(30 \times 0.8 \mathrm{~cm})$ for purification. Protein and phospholipids are estimated in each fraction obtained from the column and those with high protein to lipid ratio are pooled.

\section{Quantitative estimation of liposomes and immuno liposomes (ImLp)}

Liposome and ImLp preparation is expressed as nano or micromole Pi (inorganic phosphate)/ml after estimating their phospholipid content. ${ }^{8}$ Antibody protein in the ImLp preparation is measured by the Bradford protein assay using Bio-Rad reagents. Standard curve is plotted using BSA.

\section{Adapting ELISA for estimating antibody titre of immuno liposome}

A 96-well plate is coated with the recombinant $\mathrm{mLDHC}_{4}(8-14 \mu \mathrm{l} /$ $\mathrm{ml})$ in carbonate buffer $(0.1 \mathrm{M})$ and kept overnight at $4{ }^{\circ} \mathrm{C}$. The plate is dumped and blocked with $1 \%$ BSA in TBS for an hour at room temp followed by washing with water (MQ). Primary antibody solution is added in serial dilution of 2000, 4000, 8000, 16000 and $32000(1000 \mathrm{x})$ in triplicate wells. Liposome and ImLp each in triplicate are also added in separate wells at serial dilution of 2, 4, 8, 16 and 32 (x). Plate 
is incubated for two hours at $25^{\circ} \mathrm{C}$ before washing three times with TTBS (TBS $+1 \%$ BSA $+0.05 \%$ Tween 20 ). HRP-goat anti rabbit IgG (1:2500 dilution) is added in all the wells before incubating the plate for an hour at room temperature. After washing with TTBS and water, freshly prepared reaction mixture [ $80 \mu \mathrm{l} /$ well, citric phosphate buffer (pH $4.0,10 \mathrm{ml})$, ABTS $\left.(40 \mathrm{mM}, 0.5 \mathrm{ml}), \mathrm{H}_{2} \mathrm{O}_{2}(0.3 \%, 0.1 \mathrm{ml})\right]$ is added Color developed at different time points $(3,5,10,16$ and 20 minutes) is read at $\lambda 405 \mathrm{~nm}$ in an ELISA reader after assigning the blank wells in the program plate. The absorbance values from ImLp in triplicate are averaged after subtracting values shown by control liposome at corresponding dilutions. This is done to account for the interference caused by the lipids that constitute liposome in the immune reaction of ELISA.

\section{Quantitative estimation of appropriately oriented antibodies through ELISA titre}

Assuming the molecular weight of primary antibody as 160 $\mathrm{kDa}^{9}$ the number of antibody molecules in $\mathrm{x}$ dilution ( $\operatorname{ImLp}$ titre) is calculated. Total antibody population on ImLp is also calculated with the help of estimated protein. Quantitative assessment of correctly oriented antibodies is thus made using following formula: \% of appropriately oriented antibodies $=$ Number of antigen recognizing antibodies (calculated by concentration of antibody at corresponding $\operatorname{ImLp}$ titre $\times 2 *$ )/Total no. of antibodies incorporated in $\operatorname{ImLp}$ (calculated by estimated protein in $\operatorname{ImLp}) \times 100$. For example in I experiment (see Table 1) $0.5 \mu \mathrm{g} / \mathrm{ml}$ solution of primary antibody is prepared for ELISA out of which $100 \mu \mathrm{l} /$ well i.e. $0.05 \mu \mathrm{g}=5.0 \times$ $10^{-8} \mathrm{~g}$ antibody is used per well or moles antibody/well

$$
\frac{16 \times 10^{-6} g}{160,000}=\frac{16 \times 10^{-6} \times 10^{-4}}{16}=1 \times 10^{-10}
$$

(assuming the molecular wt as $160 \mathrm{kD}$ or $160,000 \mathrm{D}$ )

Since 1 mole contains $6.022 \times 10^{23}$ molecules (Avogadro number)

Number of antibodies $=3.125 \times 10^{-13} \times 6.022 \times 10^{23}=18.819 \times$ $10^{10}$ (at 1:1000 dilution) so the number of antibodies at 1:10 dilution (i.e. on $\operatorname{ImLp}$ ) $=18.819 \times 10^{12}=2 * \times 18.819 \times 10^{12}=37.638 \times 10^{12}$ (to account for antibodies inaccessible from top).

Total protein incorporated in $\operatorname{ImLp}(\mathrm{I})=160 \mu \mathrm{g} / \mathrm{ml}$ (see Table 1).

Since $1 \mathrm{ml}(1000 \mu \mathrm{l})$ of $\operatorname{ImLp}$ contains antibodies equivalent to $160 \mu \mathrm{g}$ protein, the $1: 10$ dilution would have $16 \mu \mathrm{g}=16 \times 10^{-6} \mathrm{~g}$ of antibodies

$$
\frac{16 \times 10^{-6} g}{160,000}=\frac{16 \times 10^{-6} \times 10^{-4}}{16}=1 \times 10^{-10} \text { moles antibodies (as per }
$$
protein estimation)

So total antibodies at $1: 10$ dilution of $\operatorname{ImLp}$ as per protein estimation is $1 \times 10^{-10} \times 6.022 \times 10^{23}=6.022 \times 10^{13}=60.22 \times 10^{12}$. However as per the titre $(1: 10)$ only $37.638 \times 10^{12}$ antibody molecules are properly oriented. Thus, $\%$ of appropriately oriented antibodies

$$
=\frac{37.638 \times 10^{12}}{60.22 \times 10^{12}} \times 100=62.501 \%
$$

*Multiplied by 2, for accounting the antibodies on the other side of liposome under the assumption that antibodies are evenly distributed over the liposome.

\section{Results}

Coupling of antibody protein on liposome surface depends on a) reaction time; b) antibody titre and c) size of liposomes. Effective dose of ImLp depends on their optimized discriminating efficiency. $\operatorname{ImLp}$ with high antibody titre would cause unwanted side effects due to antigen cross reactivity whereas lower titre may not show optimum efficiency.

Independent experiments suggest that more the antibody protein infused with the liposome, less is the likelihood of yielding appropriate orientation (Table 1). This may happen even when the liposome size is small. It is likely that the disproportionate or excessive protein causes crowding on the liposome surface that leads to compromised conformation of antibodies. Consequently, it is important to optimize the proportion of antibodies with respect to liposome size so that ImLp of desired titre can be achieved. Here titre of $\mathrm{mLDHC}_{4}$ imunoliposome is estimated with reference to primary antibody (from the same stock) taken as positive control and liposomes (without antibody) as negative control. It is observed that $\mathrm{mLDHC}_{4}$-ImLp could be detected reliably in ELISA up to 1:16 dilution (Figure 1) and the optimum incubation time for detectable color development is 15 to 20 minutes (Figure 2). Under present experimental conditions the $\mathrm{x}$ dilution of $\mathrm{ImLp}$ is in the order of 1000x of the primary antibody in terms of antigen recognition. The order of optimal dilutions i.e. the detectable range of ImLp may also vary with the size of ImLp. Apart from the method of preparation and purity of antibody it may also depend on the constituents of liposome. ImLp titre can hence be attuned within a desirable range by adjusting the size of the liposome and/or changing the liposome to antibody ratio. This is possible due to the inverse relation between the average protein accommodating ability (per unit area) and the size of the liposome.

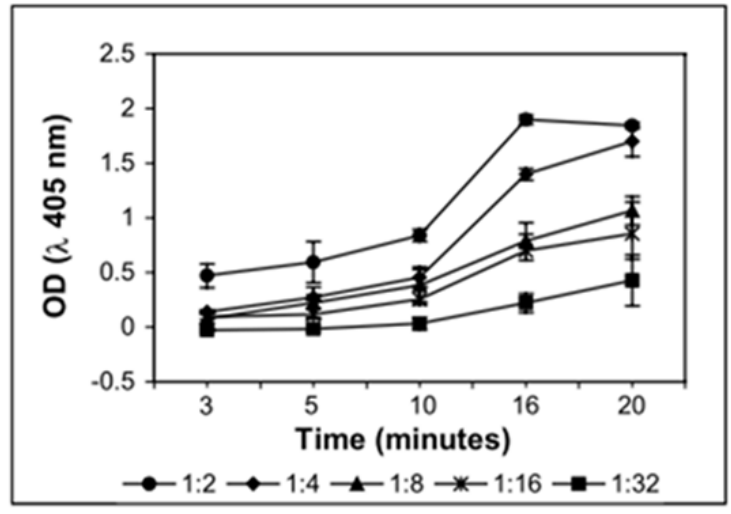

Figure I Effect of time on color development of LDHC4 -Immunoliposome (different dilutions) in i-ELISA.

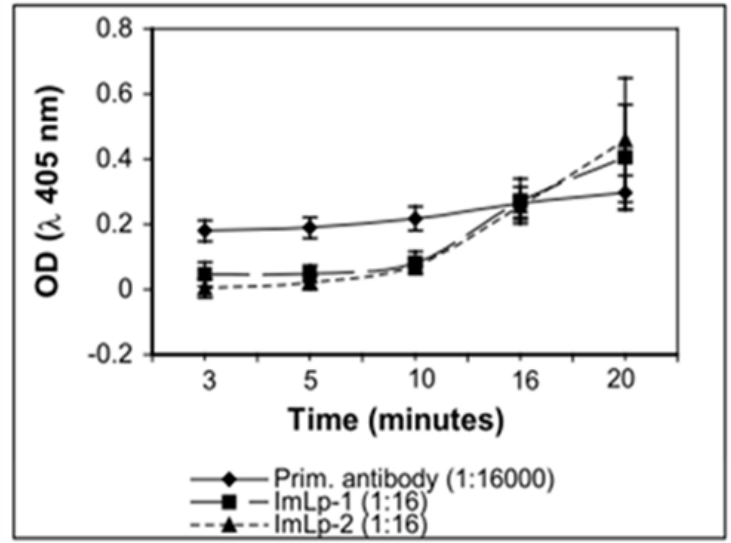

Figure 2 Antibody titre of two independent preparations of LDHC 4-Immunoliposome as measured by i- ELISA in comparison to primary antibody (RbamLDHC $\mathrm{H}_{4}$. 
Table I Clinical and biochemical variables of individuals with overweight-obesity

\begin{tabular}{llllll}
\hline $\begin{array}{l}\text { ImLp } \\
\text { Preparation }\end{array}$ & $\begin{array}{l}\text { ELISA } \\
\text { Titre of } \\
\text { ImLp }\end{array}$ & $\begin{array}{l}\text { Estimated } \\
\text { Protein in } \\
\text { ImLp }(\boldsymbol{\mu g} / \mathbf{m I})\end{array}$ & $\begin{array}{l}\text { Total no. of Antibodies } \\
\text { on ImLp Based on } \\
\text { Protein Estimation }(\mathbf{Y})\end{array}$ & $\begin{array}{l}\text { No. of Antibodies } \\
\text { Based on ImLp } \\
\text { Titre }(\mathbf{X})\end{array}$ & $\begin{array}{l}\text { Appropriately Oriented Antibodies } \\
(\%) \text { on ImLp Available for Antigen } \\
\text { Binding }(\mathbf{X} / \mathbf{Y}) \times \text { I 00 }\end{array}$ \\
\hline I & I:I0 & 160 & $60.022 \times 10^{12}$ & $18.8188 \times 10^{12}$ & $31.35 \%$ \\
II & I:I6 & 135 & $25.405 \times 10^{12}$ & $11.7617 \times 10^{12}$ & $46.30 \%$ \\
III & I:I4 & 233 & $43.848 \times 10^{12}$ & $13.4420 \times 10^{12}$ & $30.66 \%$ \\
IV & I:I6 & 300 & $56.456 \times 10^{12}$ & $11.7617 \times 10^{12}$ & $20.83 \%$ \\
\hline
\end{tabular}

$\mathrm{SD}$, standard deviation; BMI, body mass index;WC, waist circumference; AC, abdominal circumference; HC, hip circumference; RER, respiratory exchange ratio; HR, hear rate

Based on ImLp-titre, percentage of properly oriented antibody molecules could also be estimated [see inset for an example]. As an extension of ELISA, present method allows an accurate estimation of targeting efficiency of ImLps. In principle the procedure can be used for measuring titre of any ImLp, irrespective of their method of preparation and kind of antibody incorporated.

\section{Discussion}

It is important to understand that targeting efficiency needs to be defined quantitatively for the effective use of ImLp. This information is crucial to make dose related decisions and accomplish effective drug targeting. Not only their numbers but also appropriate presentation of antibodies is accountable for target discrimination. It is already established that more than optimal number of antibodies on liposomes can be detrimental ${ }^{10}$ and may lead to non-specific binding, defeating the very purpose of specific targeting for which ImLp are designed. Unlike drug-antibody conjugates, titre of antibody has no direct correlation with the titre of ImLp as the number of antibodies on each liposome depends on multiple factors including antibody purity and liposome size. In drug-antibody conjugates, which normally are stoichiometric combination, the non-interference of drug to the antigen-antibody interaction is sufficient for achieving efficient targeting. ${ }^{11}$ Whereas in case of ImLp targeting efficiency depends on antibody density which in turn depends on experimental conditions and method of its preparation. This could frequently lead to inconsistent batches in terms of targeting efficiency of ImLp and hamper its therapeutic utility. Conventionally antibodies on $\operatorname{ImL} \mathrm{p}$ are represented by protein estimation which does not reflect their conformational availability for antigen binding. This information is inadequate, also because upright orientation of antibodies cannot be ensured by general methods of preparation of ImLp. Immunoliposome or i-ELISA, described here estimates antibody titre of ImLp, which, represents the minimum dilution of ImLp that can recognize/distinguish its antigens. The information can be used for quantifying the accessibly oriented antibodies (\%) for ligand binding. It is equally valid for ImLp prepared from completely or partially purified antibodies. It also allows evaluating ImLp with other relevant antigens for cross reactivity, which can help ruling out the possible side reactions at an early stage of therapeutic development. The method described in the present study is simple, convenient and utilizes no extra equipment. It can measure and compare different batches of ImLp for better judgment with regard to therapeutic doses. Unlike the assay developed by Klegerman et al., ${ }^{12}$ which involves a specialized computer image-analysis technique for evaluating antibody-liposome conjugation efficiency present method allows to approximate targeting efficiency of ImLp that has direct therapeutic implication.

\section{Acknowledgments}

RCD thanks Prof. Goldberg for introducing her to the world of LDH proteins and also for a visiting post- doctoral fellowship.

\section{Conflicts of interest}

There is no conflict of interest.

\section{References}

1. Mastrobattista E, Koning GA, Storm G. Immunoliposomes for the targeted delivery of antitumor drugs. Adv Drug Deliv Rev. 1999;40(12):103-127.

2. Maruyama K, Ishida O, Takizawa T, et al. Possibility of active targeting to tumor tissues with liposomes. Adv Drug Deliv Rev. 1999;40(1-2): 89-102.

3. Peeters PAM, Storm G, Crommelin DJA. Immunoliposomes in vivo: state of the art. Advanced Drug Delivery Reviews. 1988;1(3):249-266.

4. Wright S, Huang L. Antibody directed liposomes as drug delivery vehicles. Advanced Drug Delivery Reviews. 1989;3(3):343-389.

5. Dutta RC. Drug carriers in pharmaceutical design: promises and progress. Curr Pharm Des. 2007;13(7):761-769.

6. Dutta RC, Goldberg E. Testis specific Lactate Dehydrogenase as target for Immunoliposomes. Am J Rep Immunol. 2008;60(1):26-32.

7. Endoh H, Suzuki Y, Hashimoto YJ. Antibody coating of liposomes with 1-ethyl-3-(3-dimethyl-aminopropyl) carbodiimide and the effect on target specificity. J Immunol Methods. 1981;44(1):79-85.

8. Ames BN, Dubin DT. The Role of Polyamines in the Neutralization of Bacteriophage Deoxyribonucleic Acid. J Biol Chem. 1960;235:769-775.

9. Kabat EA. The Molecular Weight of Antibodies. J Exp Med. 1939;69(1):103-118.

10. Houck KS, Huang L. The role of multivalency in antibody mediated liposome targeting. Biochem Biophys Res Commun. 1987;145(3):1205-1210.

11. Safavy A, Bonner JA, Waksal HW, et al. Synthesis and Biological Evaluation of Paclitaxel-C225 conjugate as a model for targeted drug delivery. Bioconjugate Chem. 2003;14(2):302-310.

12. Klegerman ME, Hamilton AJ, Huang SL, et al. Quantitative Immunoblot Assay for Assessment of Liposomal antibody conjugation Efficiency. Anal Biochem. 2002;300(1):46-52. 